Journal of Mathematics and Statistics 8 (3): 377-384, 2012

ISSN 1549-3644

(C) 2012 Science Publications

\title{
Spline Smoothing for Multi-Response Nonparametric Regression Model in Case of Heteroscedasticity of Variance
}

\author{
${ }^{1}$ Budi Lestari, ${ }^{2}$ I. Nyoman Budiantara, ${ }^{2}$ Sony Sunaryo and ${ }^{2}$ Muhammad Mashuri \\ ${ }^{1}$ Department of Mathematics, Faculty of Mathematics and Natural Sciences, \\ University of Jember, Jalan Kalimantan III/25 Kampus Tegalboto, Jember 68121, Indonesia \\ ${ }^{2}$ Department of Statistics, Faculty of Mathematics and Natural Sciences, \\ Sepuluh Nopember Institute of Technology, Surabaya, Indonesia
}

\begin{abstract}
Problem statement: Assume that data $\left(\mathrm{y}_{\mathrm{ki}}, \mathrm{t}_{\mathrm{ki}}\right), \mathrm{k}=1,2, \ldots, \mathrm{p} ; \mathrm{i}=1,2, \ldots, \mathrm{n}_{\mathrm{k}}$ where $\mathrm{n}_{\mathrm{k}}$ represents the number of repeated measurement of $\mathrm{k}^{\text {th }}$ object follows multi-response nonparametric regression model with variances of errors are heteroscedastic. Nonparametric regression curves are unknown and assumed to be smooth which are contained in Sobolev space. Random Errors are independent and normally distributed with zero means and unequal of variances. Approach: Smoothing spline can be used to estimate the nonparametric regression curve by carrying out the penalized weighted least-squares optimation. Therefore, reproducing kernel Hilbert space approach is applied to carry out the penalized weighted least-squares optimation. Results: In this study we consider the heteroscedastic multi-response nonparametric regression model and give a mathematical statistics method for obtaining the weighted spline estimator to estimate heteroscedastic multi-response nonparametric regression curves. Conclusion: The reproducing kernel Hilbert space approach gives solution of penalized weighted least-squares optimation for estimating heteroscedastic multi-response nonparametric regression curve which gives the weighted spline estimator. The estimator obtained is a biased estimator for nonparametric regression curve. However, the estimator is linear in observation.
\end{abstract}

Key words: Reproducing Kernel Hilbert Space (RKHS), Penalized Weighted Least Squares (PWLS), sobolev space, heteroscedastic, multi-response nonparametric regression

\section{INTRODUCTION}

Smoothing spline can be used to estimate functions which represent association of two or more dependent variables are observed at several values of the independent variables, such as at multiple time points. There are many researchers who have considered spline estimator for estimating regression curve of nonparametric regression model. Kimeldorf and Wahba (1971); Craven and Wahba (1979) and Wahba (1990) used original spline estimator to estimate regression curve of smooth data. Cox (1983); Cox and O'Sullivan (1996) proposed M-type spline to overcome outliers in nonparametric regression. Wahba (1983) has constructed confidence interval for original spline model by using Bayesian approach. Wahba (1985) compared between GCV and GML for choosing the smoothing parameter in the generalized spline smoothing problem. Oehlert (1992) and Koenker et al. (1994) introduced relaxed spline and quantile spline, respectively. Wang (1998) discussed smoothing spline models with correlated random errors. Wahba (2000) introduced some techniques for spline statistical model building by using reproducing kernel Hilbert spaces. Cardot et al. (2007) gave asymptotic property of smoothing splines estimators in functional linear regression with errors-in-variables. Liu et al. (2007) studied smoothing spline estimation of variance functions. Aydin (2007) showed goodness of spline estimator rather than kernel estimator in estimating nonparametric regression model for gross national product data. All these researchers studied spline estimators in case of single response nonparametric regression models only.

In the real cases, we are frequently faced the problem in which two or more dependent variables are observed at several values of the independent variables, such as at multiple time points. Multi-response nonparametric regression model provides powerful tools to model the functions which represent association of these variables. There are many researchers who have considered nonparametric models for multi-

Corresponding Author: Budi Lestari, Department of Mathematics, Faculty of Mathematics and Natural Sciences,

University of Jember, Jalan Kalimantan III/25 Kampus Tegalboto, Jember 68121, Indonesia 
response data. Wegman (1981); Miller and Wegman (1987) and Flessler (1991) proposed algorithms for spline smoothing. Wahba (1992) developed the theory of general smoothing splines using reproducing kernel Hilbert spaces. Gooijer et al. (1999) and Fernandez and Opsomer (2005) proposed methods of estimating nonparametric regression models with serially and spatially correlated errors, respectively. Wang et al. (2000) studied spline smoothing for estimating nonparametric functions from bivariate data with the same correlation of errors. Lestari et al. $(2009 ; 2010)$ studied spline estimator in multi-response nonparametric regression model with equal correlation of errors and unequal correlation of errors, respectively. Yet, all researchers discussed nonparametric regression model in case of homoscedasticity of variance.

In this study, we consider the heteroscedastic multi-response nonparametric regression model and give a mathematical statistics method for obtaining the weighted spline estimator to estimate heteroscedastic multi-response nonparametric regression curves.

\section{MATERIALS AND METHODS}

We assume that data $\left(\mathrm{y}_{\mathrm{ki}}, \mathrm{t}_{\mathrm{ki}}\right), \mathrm{k}=1,2, \ldots, \mathrm{p} ; \mathrm{i}=$ $1,2, \ldots, n_{k}$ where $n_{k}$ represents the number of repeated measurement of $\mathrm{k}^{\text {th }}$ object follows multi-response nonparametric regression model Eq. 1:

$\mathrm{y}_{\mathrm{ki}}=\mathrm{f}_{\mathrm{k}}\left(\mathrm{t}_{\mathrm{ki}}\right)+\varepsilon_{\mathrm{ki}}$

Regression curves form $f_{\mathrm{i}}, f_{2}, \ldots, f_{\mathrm{p}}$ are unknown and assumed to be smooth which are contained in Sobolev space $\mathrm{W}_{2}^{\mathrm{m}}\left[\mathrm{a}_{\mathrm{k}}, \mathrm{b}_{\mathrm{k}}\right]$. Random errors $\varepsilon_{\mathrm{ki}}$ are independent each other and normally distributed with zero means and unequal of variances, i.e., $\varepsilon_{\mathrm{ki}} \sim \underset{\text { i.j.d }}{\sim} \mathrm{N}\left(0, \sigma_{\mathrm{ki}}^{2}\right)$. Covariance matrix of random errors is given by $\left[\mathrm{W}_{\text {het }}\left(\sigma_{\sim}^{2}\right)\right]^{-1}=\operatorname{diag}\left(\mathrm{W}_{\text {het, } 1}\left(\sigma_{1}{ }^{2}\right), \ldots, \mathrm{W}_{\text {het, }}\left(\sigma_{\mathrm{p}}{ }^{2}\right)\right)$. Estimation of regression curve $\underset{\sim}{f}=\left(f_{1}, \ldots, f_{p}\right)^{T}$ can be obtained by carrying out Penalized Weighted LeastSquares (PWLS) optimation Eq. 2:

$$
\begin{aligned}
& \operatorname{Min}_{\mathrm{f}_{\mathrm{k}} \in \mathrm{W}_{2}^{\mathrm{m}}\left[a_{\mathrm{k}}, \mathrm{b}_{\mathrm{k}}\right], \mathrm{k}=1,2, \ldots, \mathrm{p}}\left\{\left(\sum_{\mathrm{k}=1}^{\mathrm{p}} \mathrm{n}_{\mathrm{k}}\right)^{-1}(\underset{\sim}{\mathrm{y}-\mathrm{f}})^{\mathrm{T}} \mathrm{W}_{\text {het }}\left({\underset{\sim}{\sigma}}^{2}\right) \times\right. \\
& \left.\underset{\sim}{(y-f)}+\sum_{k=1}^{p} \lambda_{k} \int_{a_{k}}^{b_{k}}\left[f_{k}^{\prime \prime}\left(t_{k}\right)\right]^{2} d t_{k}\right\}
\end{aligned}
$$

Reproducing Kernel Hilbert Space (RKHS) approach will give the solution of (2), i.e., weighted spline estimator that can be used to estimate heteroscedastic multi-response nonparametric regression curves.

\section{RESULTS}

Heteroscedastic multi-response nonparametric regression model: If the multi-response nonparametric regression model as shown in (1) is associated to multiresponse spline problem then for $\mathrm{i}=1,2, \ldots, \mathrm{n}_{\mathrm{k}}$ we will have model as follows Eq. 3:

$$
\left.\begin{array}{c}
\mathrm{y}_{1 \mathrm{i}}=\mathrm{L}_{\mathrm{t}_{1}} \mathrm{f}_{1}+\varepsilon_{1 \mathrm{i}} \\
\mathrm{y}_{2 \mathrm{i}}=\mathrm{L}_{\mathrm{t}_{2}} \mathrm{f}_{2}+\varepsilon_{2 \mathrm{i}} \\
\vdots \\
\mathrm{y}_{\mathrm{pi}}=\mathrm{L}_{\mathrm{t}_{\mathrm{p}}} \mathrm{f}_{\mathrm{p}}+\varepsilon_{\mathrm{pi}}
\end{array}\right\}
$$

where, $\mathrm{L}_{\mathrm{t}_{\mathrm{k}}}, \mathrm{k}=1,2, \ldots, \mathrm{p}$, represents bounded linear functional in Hilbert space $\mathrm{H}_{\mathrm{k}}$. The form of functions $f_{\mathrm{k}}, \mathrm{k}=1,2, \ldots, \mathrm{p}$ are unknown and assumed to be smooth and contained in space $\mathrm{H}_{\mathrm{k}}$. Random Errors $\varepsilon_{\mathrm{ki}}$ are independent each other and normally distributed with zero means and unequal of variances (i.e., in case of heteroscedasticity of variances). It means that

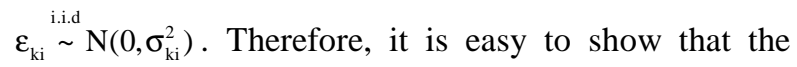
covariance matrix of random errors is given by Eq. 4 :

$$
\begin{aligned}
& {\left[\mathrm{W}_{\text {het }}\left(\sigma_{\sim}^{2}\right)\right]^{-1}=\operatorname{diag}\left(\mathrm{W}_{\text {het }, 1}\left(\sigma_{1}{ }^{2}\right), \ldots, \mathrm{W}_{\text {het, } \mathrm{p}}\left(\sigma_{\mathrm{p}}{ }^{2}\right)\right)} \\
& =\left(\begin{array}{cccc}
\mathrm{W}_{\text {het }, 1}\left(\sigma_{1}{ }^{2}\right) & 0 & \ldots & 0 \\
0 & \mathrm{~W}_{\text {het }, 2}\left(\sigma_{2}^{2}\right) & \cdots & 0 \\
\vdots & \vdots & \ddots & \vdots \\
0 & 0 & \cdots & \mathrm{W}_{\text {het, } \mathrm{p}}\left(\underset{\sim}{\left.\sigma_{\mathrm{p}}{ }^{2}\right)}\right.
\end{array}\right)
\end{aligned}
$$

where matrix $\mathrm{W}_{\text {het, } \mathrm{k}}\left(\sigma_{\mathrm{k}}{ }^{2}\right), \mathrm{k}=1,2, \ldots, \mathrm{p}$ is given by:

$$
\mathrm{W}_{\mathrm{het}, \mathrm{k}}\left(\underset{\sim}{\sigma_{\mathrm{k}}}{ }^{2}\right)=\left(\begin{array}{cccc}
\sigma_{\mathrm{k} 1}^{2} & \sigma_{\mathrm{k}(1,2)} & \cdots & \sigma_{\mathrm{k}\left(1, \mathrm{n}_{\mathrm{k}}\right)} \\
\sigma_{\mathrm{k}(2,1)} & \sigma_{\mathrm{k} 2}^{2} & \cdots & \sigma_{\mathrm{k}\left(2, \mathrm{n}_{\mathrm{k}}\right)} \\
\vdots & \vdots & \ddots & \vdots \\
\sigma_{\mathrm{k}\left(\mathrm{n}_{\mathrm{k}}, 1\right)} & \sigma_{\mathrm{k}\left(\mathrm{n}_{\mathrm{k}}, 2\right)} & \cdots & \sigma_{\mathrm{kn}}^{2}
\end{array}\right)
$$

Hilbert space $\mathrm{H}_{\mathrm{k}}$ is decomposed into direct sum of two space, i.e., space $\mathrm{N}_{k}$ and space $\mathrm{M}_{\mathrm{k}}$. So, $\mathrm{H}_{\mathrm{k}}$ can be written as:

$\mathrm{H}_{\mathrm{k}}=\mathrm{N}_{\mathrm{k}} \oplus \mathrm{M}_{\mathrm{k}}$

where, $\mathrm{N}_{\mathrm{k}}=\mathrm{M}_{\mathrm{k}}^{\perp}, \mathrm{k}=1,2, \ldots, \mathrm{p}$. Suppose that bases of space $N_{k}$ is $\left\{\theta_{k 1}, \theta_{k 2}, \ldots, \theta_{k m}\right\}$ and bases of space $M_{k}$ is $\left\{\xi_{\mathrm{k} 1}, \xi_{\mathrm{k} 2}, \ldots, \xi_{\mathrm{kn}}\right\}$, then for every $f_{\mathrm{k}} \in \mathrm{H}_{\mathrm{k}}, \mathrm{k}=1,2, \ldots, \mathrm{p}$ can be expressed as: 
$f_{k}=g_{k}+h_{k}$

where, $g_{k} \in N_{k}$ and $h_{k} \in M_{k}$. Since $\left\{\theta_{k 1}, \theta_{k 2}, \ldots, \theta_{k m}\right\}$ is bases of space $\mathrm{N}_{\mathrm{k}}$ then every function $\mathrm{g}_{\mathrm{k}} \in \mathrm{N}_{\mathrm{k}}$ can be written as Eq. 5:

$\mathrm{g}_{\mathrm{k}}=\sum_{\mathrm{v}=1}^{\mathrm{m}} \mathrm{d}_{\mathrm{kv}} \theta_{\mathrm{kv}}=\underset{\sim}{\theta_{\mathrm{k}}^{\mathrm{T}}} \mathrm{d}_{\sim}$

for any constant $\mathrm{d}_{\mathrm{kv}} \in \mathcal{R}$. Also, since $\left\{\xi_{\mathrm{k} 1}, \xi_{\mathrm{k} 2}, \ldots, \xi_{\mathrm{kn}}\right\}$ is bases of $M_{k}$ then every function $h_{k} \in M_{k}$ can be written as Eq. 6:

$\mathrm{h}_{\mathrm{k}}=\sum_{\mathrm{i}=1}^{\mathrm{n}} \mathrm{c}_{\mathrm{ki}} \xi_{\mathrm{ki}}=\underset{\sim}{\xi_{\mathrm{k}}{ }^{\mathrm{T}} \mathrm{c}_{\mathrm{k}}}$

for any constant $\mathrm{c}_{\mathrm{ki}} \in \mathcal{R}$. Therefore, for every function $f_{\mathrm{k}} \in \mathrm{H}_{\mathrm{k}}, \mathrm{k}=1,2, \ldots, \mathrm{p}$ we have Eq. 7 :

$\mathrm{f}_{\mathrm{k}}=\sum_{\mathrm{v}=1}^{\mathrm{m}} \mathrm{d}_{\mathrm{kv}} \theta_{\mathrm{kv}}+\sum_{\mathrm{i}=1}^{\mathrm{n}} \mathrm{c}_{\mathrm{ki}} \xi_{\mathrm{ki}}$

$=\theta_{\mathrm{r}}{ }^{\mathrm{T}} \mathrm{d}_{\mathrm{k}}+\boldsymbol{\xi}_{\mathrm{r}}{ }^{\mathrm{T}} \mathrm{c}_{\mathrm{k}}, \mathrm{k}=1,2, \ldots, \mathrm{p}$

where, $\quad \theta_{\mathrm{k}}=\left(\theta_{\mathrm{k} 1}, \ldots, \theta_{\mathrm{km}}\right)^{\mathrm{T}}, \quad \mathrm{d}_{\mathrm{k}}=\left(\mathrm{d}_{\mathrm{k} 1}, \ldots, \mathrm{d}_{\mathrm{km}}\right)^{\mathrm{T}}$, $\xi_{k}=\left(\xi_{k 1}, \ldots, \xi_{k n}\right)^{\mathrm{T}}$ and $c_{k}=\left(c_{k 1}, \ldots, c_{k n}\right)^{T}$. Since $L_{t_{k i}}$ represents bounded linear functional in Hilbert space $\mathrm{H}_{\mathrm{k}}$ and function $f_{\mathrm{k}} \in \mathrm{H}_{\mathrm{k}}, \mathrm{k}=1,2, \ldots, \mathrm{p}$ then we have Eq. 8:

$\mathrm{L}_{\mathrm{t}_{\mathrm{ki}}} \mathrm{f}_{\mathrm{k}}=\mathrm{L}_{\mathrm{t}_{\mathrm{i}}}\left(\mathrm{g}_{\mathrm{k}}+\mathrm{h}_{\mathrm{k}}\right)=\mathrm{L}_{\mathrm{t}_{\mathrm{ki}}}\left(\mathrm{g}_{\mathrm{k}}\right)+\mathrm{L}_{\mathrm{t}_{\mathrm{ki}}}\left(\mathrm{h}_{\mathrm{k}}\right)$

$=\mathrm{g}_{\mathrm{k}}\left(\mathrm{t}_{\mathrm{ki}}\right)+\mathrm{h}_{\mathrm{k}}\left(\mathrm{t}_{\mathrm{ki}}\right)=\mathrm{f}_{\mathrm{k}}\left(\mathrm{t}_{\mathrm{ki}}\right)$

Based on (3) and (8), the model as given in (1) can be written as follows Eq. 9:

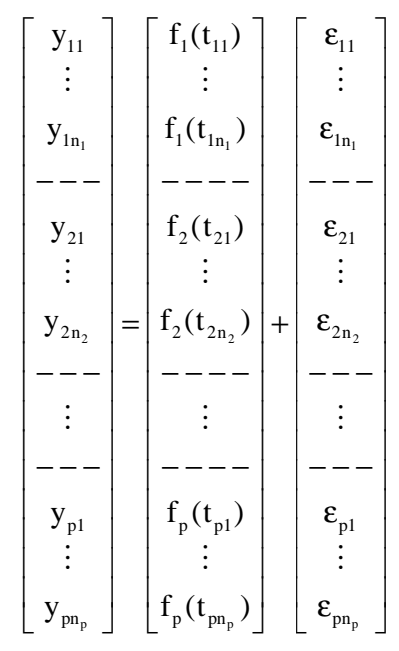

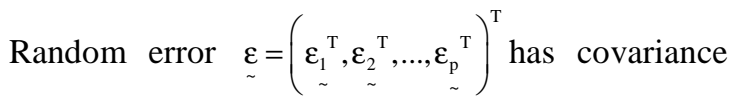
matrix as given in (4) and $\varepsilon_{\sim}=\left(\varepsilon_{\sim}^{\varepsilon_{1}}, \varepsilon_{\sim}, \ldots, \varepsilon_{\mathrm{kn}_{\mathrm{k}}}\right)^{\mathrm{T}}, \mathrm{k}=$ $1,2, \ldots, \mathrm{p}$

Weighted spline estimator for estimating heteroscedastic multi-response nonparametric regression curve: Recall the heteroscedastic nonparametric regression model given in (9). By considering Riesz representation theorem (Wahba, 1992) and $L_{t_{k i}}$ is bounded linear functional in space $H_{k}$ then there exists $\eta_{k i} \in H_{k}$ that is to be representer of $\mathrm{L}_{\mathrm{t}_{\mathrm{ki}}}$ and satisfies Eq. 10:

$\mathrm{L}_{\mathrm{t}_{\mathrm{ki}}} \mathrm{f}_{\mathrm{k}}=\left\langle\eta_{\mathrm{ki}}, \mathrm{f}_{\mathrm{k}}\right\rangle=\mathrm{f}_{\mathrm{k}}\left(\mathrm{t}_{\mathrm{ki}}\right), \mathrm{f}_{\mathrm{k}} \in \mathrm{H}_{\mathrm{k}}$

where, $\langle. .$,$\rangle represents an inner product. Because of (7)$ and by considering inner product properties, we have:

$$
\begin{aligned}
& \mathrm{f}_{\mathrm{k}}\left(\mathrm{t}_{\mathrm{ki}}\right)=\left\langle\eta_{\mathrm{ki}}, \theta_{\mathrm{k}}{ }^{\mathrm{T}} \mathrm{d}_{\mathrm{k}}+\xi_{\mathrm{k}}{ }^{\mathrm{T}} \mathrm{c}_{\mathrm{k}}\right\rangle \\
& =\left\langle\eta_{k i}, \theta^{{ }_{k}{ }^{T}} d_{k}\right\rangle+\left\langle\eta_{k i}, \xi_{k}{ }^{T} c_{k}\right\rangle
\end{aligned}
$$

Furthermore, for $\mathrm{k}=1$ we have:

$\mathrm{f}_{1}\left(\mathrm{t}_{\mathrm{li}}\right)=\left\langle\eta_{1 \mathrm{i}}, \theta_{\sim}^{\mathrm{T}} \mathrm{d}_{1}\right\rangle+\left\langle\eta_{1 \mathrm{i}}, \xi_{\sim}^{\mathrm{T}} \mathrm{c}_{\sim}\right\rangle, \mathrm{i}=1,2, \ldots, \mathrm{n}_{1}$

Therefore, for $\mathrm{i}=1$ we obtain Eq. 11:

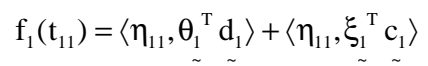

$=\left\langle\eta_{11},\left(\begin{array}{llll}\theta_{11} & \theta_{12} & \cdots & \theta_{1 \mathrm{~m}_{1}}\end{array}\right)\left(\begin{array}{c}\mathrm{d}_{11} \\ d_{12} \\ \vdots \\ d_{\mathrm{m}_{1}}\end{array}\right)\right\rangle+$

$\left\langle\eta_{11},\left(\begin{array}{llll}\xi_{11} & \xi_{12} & \cdots & \xi_{1 n_{1}}\end{array}\right)\left(\begin{array}{c}c_{11} \\ c_{12} \\ \vdots \\ c_{1 n_{1}}\end{array}\right)\right\rangle$

$=\mathrm{d}_{11}\left\langle\eta_{11}, \theta_{11}\right\rangle+\mathrm{d}_{12}\left\langle\eta_{11}, \theta_{12}\right\rangle+\ldots+$

$\mathrm{d}_{1 \mathrm{~m}_{1}}\left\langle\eta_{11}, \theta_{1 \mathrm{~m}_{1}}\right\rangle+\mathrm{c}_{11}\left\langle\eta_{11}, \xi_{11}\right\rangle+$

$c_{12}\left\langle\eta_{11}, \xi_{12}\right\rangle+\ldots+c_{1 n_{1}}\left\langle\eta_{1 n_{1}}, \xi_{1 n_{1}}\right\rangle$

If the process to be continued for $\mathrm{i}=\mathrm{n}_{1}$ in the similar way, we obtain Eq. 12: 
$\mathrm{f}_{1}\left(\mathrm{t}_{\mathrm{In}_{1}}\right)=\mathrm{d}_{11}\left\langle\eta_{\mathrm{In}_{1}}, \theta_{11}\right\rangle+\mathrm{d}_{12}\left\langle\eta_{\mathrm{In}_{1}}, \theta_{12}\right\rangle+\ldots+$

$\mathrm{d}_{\mathrm{Im}_{1}}\left\langle\eta_{\mathrm{In}_{1}}, \theta_{1 \mathrm{~m}_{1}}\right\rangle+\mathrm{c}_{11}\left\langle\eta_{\mathrm{In}_{1}}, \xi_{11}\right\rangle+$

$c_{12}\left\langle\eta_{1 n_{1}}, \xi_{12}\right\rangle+\ldots+c_{1 n_{1}}\left\langle\eta_{1 n_{1}}, \xi_{1 n_{1}}\right\rangle$

Based on both (11) and (12), vector $f_{1}\left(t_{1}\right)$ can be represented as Eq. 13:

$$
\begin{aligned}
& \mathrm{f}_{1}\left(\mathrm{t}_{1}\right)=\left[\begin{array}{c}
\mathrm{f}_{1}\left(\mathrm{t}_{11}\right) \\
\mathrm{f}_{1}\left(\mathrm{t}_{12}\right) \\
\vdots \\
\mathrm{f}_{1}\left(\mathrm{t}_{1 \mathrm{n}_{1}}\right)
\end{array}\right] \\
& =\left[\begin{array}{cccc}
\left\langle\eta_{11}, \theta_{11}\right\rangle & \left\langle\eta_{11}, \theta_{12}\right\rangle & \cdots & \left\langle\eta_{11}, \theta_{1 m_{1}}\right\rangle \\
\left\langle\eta_{12}, \theta_{11}\right\rangle & \left\langle\eta_{12}, \theta_{12}\right\rangle & \cdots & \left\langle\eta_{12}, \theta_{1 m_{1}}\right\rangle \\
\vdots & \vdots & \vdots & \vdots \\
\left\langle\eta_{1 n_{1}}, \theta_{11}\right\rangle & \left\langle\eta_{1 n_{1}}, \theta_{12}\right\rangle & \cdots & \left\langle\eta_{1 n_{1}}, \theta_{1 m_{1}}\right\rangle
\end{array}\right] \times \\
& {\left[\begin{array}{c}
\mathrm{d}_{11} \\
\mathrm{~d}_{12} \\
\vdots \\
\mathrm{d}_{1 \mathrm{~m}_{1}}
\end{array}\right]+} \\
& {\left[\begin{array}{cccc}
\left\langle\eta_{11}, \xi_{11}\right\rangle & \left\langle\eta_{11}, \xi_{12}\right\rangle & \cdots & \left\langle\eta_{11}, \xi_{1 n_{1}}\right\rangle \\
\left\langle\eta_{12}, \xi_{11}\right\rangle & \left\langle\eta_{12}, \xi_{12}\right\rangle & \cdots & \left\langle\eta_{12}, \xi_{1 n_{1}}\right\rangle \\
\vdots & \vdots & \vdots & \vdots \\
\left\langle\eta_{1 n_{1}}, \xi_{11}\right\rangle & \left\langle\eta_{1 n_{1}}, \xi_{12}\right\rangle & \cdots & \left\langle\eta_{1 n_{1}}, \xi_{1 n_{1}}\right\rangle
\end{array}\right]\left[\begin{array}{c}
c_{11} \\
c_{12} \\
\vdots \\
c_{1 n_{1}}
\end{array}\right]} \\
& =\mathrm{T}_{1} \mathrm{~d}_{1}+\mathrm{V}_{1} \mathrm{c}_{1}
\end{aligned}
$$

Where:

$$
\begin{aligned}
& \mathrm{T}_{1}=\left[\begin{array}{cccc}
\left\langle\eta_{11}, \theta_{11}\right\rangle & \left\langle\eta_{11}, \theta_{12}\right\rangle & \cdots & \left\langle\eta_{11}, \theta_{1 \mathrm{~m}_{1}}\right\rangle \\
\left\langle\eta_{12}, \theta_{11}\right\rangle & \left\langle\eta_{12}, \theta_{12}\right\rangle & \cdots & \left\langle\eta_{12}, \theta_{1 \mathrm{~m}_{1}}\right\rangle \\
\vdots & \vdots & \vdots & \vdots \\
\left\langle\eta_{1 n_{1}}, \theta_{11}\right\rangle & \left\langle\eta_{1 n_{1}}, \theta_{12}\right\rangle & \cdots & \left\langle\eta_{1 n_{1}}, \theta_{1 \mathrm{l}_{1}}\right\rangle
\end{array}\right] \\
& V_{1}=\left[\begin{array}{cccc}
\left\langle\eta_{11}, \xi_{11}\right\rangle & \left\langle\eta_{11}, \xi_{12}\right\rangle & \cdots & \left\langle\eta_{11}, \xi_{1 n_{1}}\right\rangle \\
\left\langle\eta_{12}, \xi_{11}\right\rangle & \left\langle\eta_{12}, \xi_{12}\right\rangle & \cdots & \left\langle\eta_{12}, \xi_{1 n_{1}}\right\rangle \\
\vdots & \vdots & \vdots & \vdots \\
\left\langle\eta_{1 n_{1}}, \xi_{11}\right\rangle & \left\langle\eta_{1 n_{1}}, \xi_{12}\right\rangle & \cdots & \left\langle\eta_{1 n_{1}}, \xi_{1 n_{1}}\right\rangle
\end{array}\right] \\
& \underset{\sim}{\mathrm{d}_{1}}=\left[\begin{array}{c}
\mathrm{d}_{11} \\
\mathrm{~d}_{12} \\
\vdots \\
\mathrm{d}_{1 \mathrm{~m}_{1}}
\end{array}\right] \underset{\sim}{\text { and } \mathrm{c}_{1}}=\left[\begin{array}{c}
\mathrm{c}_{11} \\
\mathrm{c}_{12} \\
\vdots \\
\mathrm{c}_{1 \mathrm{n}_{1}}
\end{array}\right]
\end{aligned}
$$

Since $\left\langle\eta_{1 \mathrm{li}}, \xi_{1 \mathrm{k}}\right\rangle=\left\langle\xi_{\mathrm{li}}, \xi_{\mathrm{lk}}\right\rangle$ then $\mathrm{V}_{1}$ can be written as follows Eq. 14:
$\mathrm{V}_{1}=\left[\begin{array}{cccc}\left\langle\xi_{11}, \xi_{11}\right\rangle & \left\langle\xi_{11}, \xi_{12}\right\rangle & \cdots & \left\langle\xi_{11}, \xi_{1 n_{1}}\right\rangle \\ \left\langle\xi_{12}, \xi_{11}\right\rangle & \left\langle\xi_{12}, \xi_{12}\right\rangle & \cdots & \left\langle\xi_{12}, \xi_{1 n_{1}}\right\rangle \\ \vdots & \vdots & \vdots & \vdots \\ \left\langle\xi_{1 n_{1}}, \xi_{11}\right\rangle & \left\langle\xi_{1 n_{1}}, \xi_{12}\right\rangle & \cdots & \left\langle\xi_{1 n_{1}}, \xi_{1 n_{1}}\right\rangle\end{array}\right]$

Similarly, we get: $\mathrm{f}_{2}\left(\mathrm{t}_{2}\right)=\mathrm{T}_{2} \mathrm{~d}_{2}+\mathrm{V}_{2} \mathrm{c}_{2}, \ldots$, $f_{p}\left(t_{p}\right)=T_{p} d_{p}+V_{p} c_{p}$. Therefore, regression function $\underset{\sim}{f}(t)$ can be represented as follows Eq. 15:

$$
\begin{aligned}
& \underset{\sim}{\mathrm{f}}(\mathrm{t})=\left[\begin{array}{c}
\mathrm{f}_{1}\left(\mathrm{t}_{1}\right) \\
\sim \\
\mathrm{f}_{2}\left(\mathrm{t}_{2}\right) \\
\sim \\
\vdots \\
\mathrm{f}_{\mathrm{p}}\left(\mathrm{t}_{\mathrm{p}}\right)
\end{array}\right]=\left[\begin{array}{c}
\mathrm{T}_{1} \mathrm{~d}_{1} \\
\sim \\
\mathrm{T}_{2} \mathrm{~d}_{2} \\
\vdots \\
\vdots \\
\mathrm{T}_{\mathrm{p}} \mathrm{d}_{\mathrm{p}}
\end{array}\right]+\left[\begin{array}{c}
\mathrm{V}_{1} \mathrm{c}_{1} \\
\sim \\
\mathrm{V}_{2} \mathrm{c}_{2} \\
\vdots \\
\sim \\
\mathrm{V}_{\mathrm{p}} \mathrm{c}_{\mathrm{p}}
\end{array}\right] \\
& =\left[\begin{array}{cccc}
\mathrm{T}_{1} & 0 & \cdots & 0 \\
0 & \mathrm{~T}_{2} & \cdots & 0 \\
\vdots & \vdots & \ddots & \vdots \\
0 & 0 & \cdots & \mathrm{T}_{\mathrm{p}}
\end{array}\right]\left[\begin{array}{c}
\mathrm{d}_{1} \\
\tilde{c} \\
\mathrm{~d}_{2} \\
\tilde{\vdots} \\
\mathrm{d}_{\mathrm{p}} \\
\sim
\end{array}\right]+ \\
& {\left[\begin{array}{cccc}
\mathrm{V}_{1} & 0 & \cdots & 0 \\
0 & \mathrm{~V}_{2} & \cdots & 0 \\
\vdots & \vdots & \ddots & \vdots \\
0 & 0 & \cdots & \mathrm{V}_{\mathrm{p}}
\end{array}\right]\left[\begin{array}{c}
\mathrm{c}_{1} \\
\sim \\
\mathrm{c}_{2} \\
2 \\
\vdots \\
\mathrm{c}_{\mathrm{p}} \\
\sim
\end{array}\right]} \\
& =\underset{\sim}{\mathrm{d}}+\mathrm{V}_{\sim}^{\mathrm{c}}
\end{aligned}
$$

In (15), $\mathrm{T}$ is a $(\mathrm{N} \times \mathrm{M})$ matrix where $\mathrm{N}=\sum_{\mathrm{k}=1}^{\mathrm{p}} \mathrm{n}_{\mathrm{k}}$, $\mathrm{M}=\sum_{\mathrm{k}=1}^{\mathrm{p}} \mathrm{m}_{\mathrm{k}}$ and $\underset{\sim}{\mathrm{d}}$ is a $(\mathrm{M} \times 1)$ vector of parameters which are given by:

$\mathrm{T}=\left[\begin{array}{cccc}\mathrm{T}_{1} & 0 & \cdots & 0 \\ 0 & \mathrm{~T}_{2} & \cdots & 0 \\ \vdots & \vdots & \ddots & \vdots \\ 0 & 0 & \cdots & \mathrm{T}_{\mathrm{p}}\end{array}\right]$ and $\underset{\sim}{\mathrm{d}}=\left[\begin{array}{c}\mathrm{d}_{1}{ }^{\mathrm{T}} \\ \mathrm{d}_{2} \\ \underset{\sim}{\mathrm{T}} \\ \vdots \\ \mathrm{d}_{\sim}{ }^{\mathrm{T}}\end{array}\right]$

respectively. Also, $\mathrm{V}$ is a $(\mathrm{N} \times \mathrm{N})$ matrix and $\mathrm{c}$ is a $(\mathrm{N}$ $\times 1)$ vector of parameters given by: 
$\mathrm{V}=\left[\begin{array}{cccc}\mathrm{V}_{1} & 0 & \cdots & 0 \\ 0 & \mathrm{~V}_{2} & \cdots & 0 \\ \vdots & \vdots & \ddots & \vdots \\ 0 & 0 & \cdots & \mathrm{V}_{\mathrm{p}}\end{array}\right]$ and $\underset{\sim}{\mathrm{c}}=\left[\begin{array}{c}\mathrm{c}_{1}{ }^{\mathrm{T}} \\ \underset{\sim}{\mathrm{c}_{2}} \\ \sim \\ \vdots \\ \mathrm{c}_{\mathrm{p}}{ }^{\mathrm{T}} \\ \sim\end{array}\right]$

respectively. Therefore, the heteroscedastic multiresponse nonparametric regression model as given in (1) can be written as follows:

$\mathrm{y}=\mathrm{T} \underset{\sim}{\mathrm{d}}+\mathrm{V} \underset{\sim}{\mathrm{c}}+\underset{\sim}{\varepsilon}$

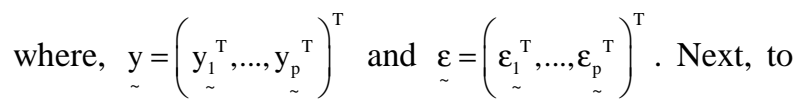
obtain estimate regression curve $\underset{\sim}{f}$, we use RKHS approach by solving the weighted optimation Eq. 16:

$$
\begin{aligned}
& \operatorname{Min}_{\substack{\mathrm{f}_{\mathrm{k}} \in \mathrm{H}_{\mathrm{k}} \\
\mathrm{k}=1,2, \ldots, \mathrm{p}}}\left\{\left\|\mathrm{W}_{\mathrm{het}}^{\frac{1}{2}}\left(\underset{\sim}{\sigma^{2}}\right) \underset{\sim}{\varepsilon}\right\|^{2}\right\} \\
& =\operatorname{Min}_{\substack{\mathrm{f}_{\mathrm{k}} \in \mathrm{H}_{\mathrm{k}} \\
\mathrm{k}=1,2, \ldots, \mathrm{p}}}\left\{\left\|\mathrm{W}_{\mathrm{het}}^{\frac{1}{2}}\left(\underset{\sim}{\sigma^{2}}\right) \underset{\sim}{(\mathrm{y}-\underset{\sim}{\mathrm{f}})}\right\|^{2}\right\}
\end{aligned}
$$

with constrain Eq. 17:

$$
\left\|\mathrm{f}_{\mathrm{k}}\right\|^{2}<\gamma_{\mathrm{k}}, \gamma_{\mathrm{k}} \geq 0, \mathrm{k}=1,2, \ldots, \mathrm{p}
$$

Let $\mathrm{H}_{\mathrm{k}}=\mathrm{W}_{2}^{\mathrm{m}}\left[\mathrm{a}_{\mathrm{k}}, \mathrm{b}_{\mathrm{k}}\right]$ where $\mathrm{W}_{2}^{\mathrm{m}}\left[\mathrm{a}_{\mathrm{k}}, \mathrm{b}_{\mathrm{k}}\right]$ is a $2^{\mathrm{nd}}-$ order Sobolev space given by:

$$
\mathrm{W}_{2}^{\mathrm{m}}\left[\mathrm{a}_{\mathrm{k}}, \mathrm{b}_{\mathrm{k}}\right]=\left\{\mathrm{f} ; \int_{\mathrm{a}_{\mathrm{k}}}^{\mathrm{b}_{\mathrm{k}}}\left[\mathrm{f}_{\mathrm{k}}^{(\mathrm{m})}\left(\mathrm{t}_{\mathrm{k}}\right) \mathrm{dt}_{\mathrm{k}}<\infty\right\}\right.
$$

where, $a_{k} \leq t_{k} \leq b_{k}, k=1,2, \ldots, p$. Based on space $\mathrm{W}_{2}^{\mathrm{m}}\left[\mathrm{a}_{\mathrm{k}}, \mathrm{b}_{\mathrm{k}}\right]$, for every function $\mathrm{f}_{\mathrm{k}} \in \mathrm{W}_{2}^{\mathrm{m}}\left[\mathrm{a}_{\mathrm{k}}, \mathrm{b}_{\mathrm{k}}\right]$ we define norm as follows:

$$
\left\|f_{k}\right\|^{2}=\int_{a_{k}}^{b_{k}}\left[f_{k}^{(m)}\left(t_{k}\right)\right]^{2} d t_{k}, k=1,2, \ldots, p
$$

Therefore, the weighted optimation in (16) with constrain in (17) can be written as Eq. 18:

$$
\operatorname{Min}_{\substack{\mathrm{f}_{\mathrm{k}} \in \mathrm{H}_{\mathrm{k}} \\ \mathrm{k}=1,2, \ldots, \mathrm{p}}}\left\{\left\|\mathrm{W}_{\mathrm{het}}^{\frac{1}{2}}\left(\underset{\sim}{\sigma^{2}}\right) \underset{\sim}{\varepsilon}\right\|^{2}\right\}=\operatorname{Min}_{\substack{\mathrm{f}_{\mathrm{k}} \in \mathrm{H}_{\mathrm{k}} \\ \mathrm{k}=1,2, \ldots, \mathrm{p}}}\left\{\| \mathrm{W}_{\mathrm{het}}^{\frac{1}{2}}\left(\underset{\sim}{\sigma^{2}}\right)\left(\underset{\sim}{\mathrm{y}-\mathrm{f})}\left\|^{2}\right\|^{2}\right\}\right.
$$

with constrain Eq. 19:

$$
\int_{\mathrm{a}_{\mathrm{k}}}^{\mathrm{b}_{\mathrm{k}}}\left[\mathrm{f}_{\mathrm{k}}^{(\mathrm{m})}\left(\mathrm{t}_{\mathrm{k}}\right)\right]^{2} \mathrm{dt}_{\mathrm{k}}<\gamma_{\mathrm{k}}, \gamma_{\mathrm{k}} \geq 0
$$

The weighted optimation (18) with constrain (19) is equivalent to solve the PWLS optimation Eq. 20:

$$
\begin{aligned}
& \operatorname{Min}_{\substack{\mathrm{f}_{\mathrm{k}} \in \mathrm{W}_{2}^{\mathrm{m}}\left[\mathrm{a}_{\mathrm{k}}, \mathrm{b}_{\mathrm{k}}\right] \\
\mathrm{k}=1,2, \ldots, \mathrm{p}}}\left\{\mathrm{N}^{-1}(\underset{\sim}{\mathrm{y}-\mathrm{f}})^{\mathrm{T}} \mathrm{W}_{\text {het }}\left(\underset{\sim}{\sigma^{2}}\right)(\underset{\sim}{\mathrm{y}}-\underset{\sim}{\mathrm{f}})+\right. \\
& \left.\sum_{\mathrm{k}=1}^{\mathrm{p}} \lambda_{\mathrm{k}} \int_{\mathrm{a}_{\mathrm{k}}}^{\mathrm{b}_{\mathrm{k}}}\left[\mathrm{f}_{\mathrm{k}}^{(\mathrm{m})}\left(\mathrm{t}_{\mathrm{k}}\right)\right]^{2} \mathrm{dt}_{\mathrm{k}}\right\}
\end{aligned}
$$

where, $\lambda_{\mathrm{k}}, \mathrm{k}=1,2, \ldots, \mathrm{p}$ are smoothing parameters which control between goodness of fit:

$\mathrm{N}^{-1}(\mathrm{y}-\mathrm{f})^{\mathrm{T}} \mathrm{W}_{\text {het }}\left(\sigma^{2}\right)(\mathrm{y}-\mathrm{f})$

and penalty:

$$
\lambda_{1} \int_{a_{1}}^{b_{1}}\left[f_{1}^{(m)}\left(t_{1}\right)\right]^{2} d t_{1}+\ldots+\lambda_{p} \int_{a_{p}}^{b_{p}}\left[f_{p}^{(m)}\left(t_{p}\right)\right]^{2} d t_{p}
$$

To obtain the solution of (20), firstly, we decompose penalty component as follows:

$$
\begin{aligned}
& \lambda_{1} \int_{\mathrm{a}_{1}}^{\mathrm{b}_{1}}\left[\mathrm{f}_{1}^{(\mathrm{m})}\left(\mathrm{t}_{1}\right)\right]^{2} \mathrm{dt}_{1}=\left\|\mathrm{Pf}_{1}\right\|^{2}=\left\langle\mathrm{Pf}_{1}, \mathrm{Pf}_{1}\right\rangle
\end{aligned}
$$

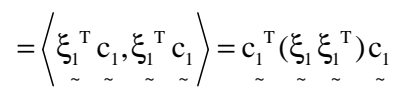

$$
\begin{aligned}
& =\left(\begin{array}{llll}
\mathrm{c}_{11} & \mathrm{c}_{12} & \cdots & \mathrm{c}_{1 \mathrm{n}_{1}}
\end{array}\right) \times \\
& \left(\begin{array}{cccc}
\left\langle\xi_{11}, \xi_{11}\right\rangle & \left\langle\xi_{11}, \xi_{12}\right\rangle & \cdots & \left\langle\xi_{11}, \xi_{1 n_{1}}\right\rangle \\
\left\langle\xi_{12}, \xi_{11}\right\rangle & \left\langle\xi_{12}, \xi_{12}\right\rangle & \cdots & \left\langle\xi_{12}, \xi_{1 n_{1}}\right\rangle \\
\vdots & \vdots & \vdots & \vdots \\
\left\langle\xi_{1 n_{1}}, \xi_{11}\right\rangle & \left\langle\xi_{1 n_{1}}, \xi_{12}\right\rangle & \cdots & \left\langle\xi_{1 n_{1}}, \xi_{1 n_{1}}\right\rangle
\end{array}\right) \times \\
& \left(\begin{array}{c}
\mathrm{c}_{11} \\
\mathrm{c}_{12} \\
\vdots \\
\mathrm{c}_{1 \mathrm{n}_{1}}
\end{array}\right)=\underset{\sim}{\mathrm{c}_{1}}{ }^{\mathrm{T}} \mathrm{V}_{1} \mathrm{c}_{1}
\end{aligned}
$$

Consequently, we have Eq. 21:

$$
\lambda_{1} \int_{\mathrm{a}_{1}}^{\mathrm{b}_{1}}\left[\mathrm{f}_{1}^{(\mathrm{m})}\left(\mathrm{t}_{1}\right)\right]^{2} \mathrm{dt}_{1}=\lambda_{1} \mathrm{c}_{\sim}^{\mathrm{T}} \mathrm{V}_{1} \mathrm{c}_{\sim}
$$

Similarly, we obtain:

$$
\begin{gathered}
\lambda_{2} \int_{\mathrm{a}_{2}}^{\mathrm{b}_{2}}\left[\mathrm{f}_{2}^{(\mathrm{m})}\left(\mathrm{t}_{2}\right)\right]^{2} \mathrm{dt}_{2}=\lambda_{2}{\underset{\sim}{\mathrm{c}_{2}}{ }^{\mathrm{T}} \mathrm{V}_{2} \underset{\sim}{\mathrm{c}_{2}}}_{\vdots} \\
\lambda_{\mathrm{p}} \int_{\mathrm{a}_{\mathrm{p}}}^{\mathrm{b}_{\mathrm{p}}}\left[\mathrm{f}_{\mathrm{p}}^{(\mathrm{m})}\left(\mathrm{t}_{\mathrm{p}}\right)\right]^{2} \mathrm{dt}_{\mathrm{p}}=\lambda_{\mathrm{p}} \underset{\sim}{\mathrm{c}_{\mathrm{p}}{ }^{\mathrm{T}} \mathrm{V}_{\mathrm{p}}{\underset{\sim}{\mathrm{p}}}_{\mathrm{p}}}
\end{gathered}
$$


Based on (21) and (22), we have the penalty Eq. 23:

$$
\begin{aligned}
& \left.\sum_{\mathrm{k}=1}^{\mathrm{p}} \lambda_{\mathrm{k}} \int_{\mathrm{a}_{\mathrm{k}}}^{\mathrm{b}_{\mathrm{k}}}\left[\mathrm{f}_{\mathrm{k}}^{(\mathrm{m})}\left(\mathrm{t}_{\mathrm{k}}\right)\right]^{2} \mathrm{dt}_{\mathrm{k}}\right\} \\
& =\left(\begin{array}{cccc}
\mathrm{c}_{1}{ }^{\mathrm{T}} & \mathrm{c}_{2}{ }^{\mathrm{T}} & \cdots & \mathrm{c}_{\mathrm{p}}{ }^{\mathrm{T}}
\end{array}\right) \times \\
& \left(\begin{array}{cccc}
\lambda_{1} \mathrm{I}_{\mathrm{n}_{1}} & 0 & \cdots & 0 \\
0 & \lambda_{2} \mathrm{I}_{\mathrm{n}_{2}} & \cdots & 0 \\
\vdots & \vdots & \ddots & \vdots \\
0 & 0 & \cdots & \lambda_{\mathrm{p}} \mathrm{I}_{\mathrm{n}_{\mathrm{p}}}
\end{array}\right) \times \\
& \left(\begin{array}{cccc}
\mathrm{V}_{1} & 0 & \cdots & 0 \\
0 & \mathrm{~V}_{2} & \cdots & 0 \\
\vdots & \vdots & \ddots & \vdots \\
0 & 0 & \cdots & \mathrm{V}_{\mathrm{p}}
\end{array}\right)\left(\begin{array}{c}
\mathrm{c}_{1} \\
\sim \\
\mathrm{c}_{2} \\
\vdots \\
\mathrm{c}_{\mathrm{p}} \\
\sim
\end{array}\right)=\mathrm{c}_{\sim}^{\mathrm{T}} \lambda \mathrm{V}_{\sim}^{\mathrm{c}}
\end{aligned}
$$

where, $\lambda=\operatorname{diag}\left(\lambda_{1} \mathrm{I}_{\mathrm{n}_{1}}, \lambda_{2} \mathrm{I}_{\mathrm{n}_{2}}, \ldots, \lambda_{\mathrm{p}} \mathrm{I}_{\mathrm{n}_{\mathrm{p}}}\right)$. The goodness of fit in PWLS given in (20) can be written as:

$\mathrm{N}^{-1}(\mathrm{y}-\mathrm{f})^{\mathrm{T}} \mathrm{W}_{\text {het }}\left(\sigma_{\sim}^{2}\right)(\mathrm{y}-\mathrm{f})$

$=\mathrm{N}^{-1}(\mathrm{y}-\mathrm{Td}-\mathrm{Vc})^{\mathrm{T}} \mathrm{W}_{\text {het }}\left(\sigma^{2}\right)(\mathrm{y}-\mathrm{Td}-\mathrm{Vc})$

Next, if we combine between goodness of fit and penalty, we get PWLS optimation as follows Eq. 24:

$$
\begin{aligned}
& \operatorname{Min}_{\substack{\mathrm{f}_{\mathrm{k}} \in \mathrm{W}^{\mathrm{m}}\left[\mathrm{W}_{\mathrm{k}}, b_{\mathrm{k}}\right] \\
\mathrm{k}=1,2, \ldots, \ldots,}}\left\{\mathrm{N}^{-1}(\underset{\sim}{\mathrm{y}}-\underset{\sim}{\mathrm{f}})^{\mathrm{T}} \mathrm{W}_{\text {het }}\left(\underset{\sim}{\sigma^{2}}\right)(\underset{\sim}{\mathrm{y}-\mathrm{f}})+\right. \\
& \left.\sum_{\mathrm{k}=1}^{\mathrm{p}} \lambda_{\mathrm{k}} \int_{\mathrm{a}_{\mathrm{k}}}^{\mathrm{b}_{\mathrm{k}}}\left[\mathrm{f}_{\mathrm{k}}^{(\mathrm{m})}\left(\mathrm{t}_{\mathrm{k}}\right)\right]^{2} \mathrm{dt}_{\mathrm{k}}\right\} \\
& =\underset{\substack{c \in R^{p n} \\
d \in R^{p m}}}{\operatorname{Min}}\left\{\left(\underset{\sim}{\mathrm{y}}-\underset{\sim}{\mathrm{T}}-\mathrm{V}_{\sim}^{\mathrm{c}}\right)^{\mathrm{T}} \mathrm{W}_{\text {het }}\left(\underset{\sim}{\sigma^{2}}\right) \times\right. \\
& \left.(\mathrm{y}-\mathrm{Td}-\underset{\sim}{\mathrm{d}} \mathrm{c})+\mathrm{c}_{\sim}^{\mathrm{T}} \mathrm{N} \lambda \mathrm{Vc}\right\}
\end{aligned}
$$

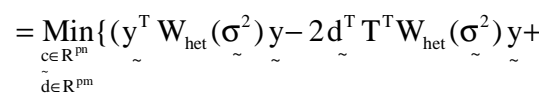

$$
\begin{aligned}
& -2 \mathrm{c}_{\sim}^{\mathrm{T}} \mathrm{V}^{\mathrm{T}} \mathrm{W}_{\text {het }}\left(\underset{\sim}{\sigma^{2}}\right) \mathrm{y}+\underset{\sim}{\mathrm{d}^{\mathrm{T}}} \mathrm{T}^{\mathrm{T}} \mathrm{W}_{\text {het }}\left(\underset{\sim}{\sigma^{2}}\right) \mathrm{Td}+ \\
& \mathrm{d}_{\sim}^{\mathrm{T}} \mathrm{T}^{\mathrm{T}} \mathrm{W}_{\text {het }}\left(\sigma_{\sim}^{2}\right) \mathrm{V} \mathrm{c}+\mathrm{c}_{\sim}^{\mathrm{T}} \mathrm{V}^{\mathrm{T}} \mathrm{W}_{\text {het }}\left(\sigma_{\sim}^{2}\right) \mathrm{Td}+ \\
& \mathrm{c}_{\sim}^{\mathrm{T}}\left(\mathrm{V}^{\mathrm{T}} \mathrm{W}_{\text {het }}\left(\sigma_{\sim}^{2}\right) \mathrm{V}+\mathrm{N} \lambda \mathrm{V}\right) \underset{\sim}{\mathrm{c}\}} \\
& =\underset{\substack{\mathrm{c} \in \mathrm{R}^{\mathrm{pn}} \\
\mathrm{d} \in \mathrm{R}^{\mathrm{pm}}}}{\operatorname{Min}}\{\underset{\sim}{\mathrm{c}, \mathrm{d})}\}
\end{aligned}
$$

The solution of optimation (24) can be obtained by taking partially derivative of $\mathrm{Q}(\underset{\sim}{\mathrm{c}, \mathrm{d})}$ with respect to $\mathrm{c}$ and $\mathrm{d}$ and then the results are to be equal to zero. Therefore, we have partially derivative Eq. 25:

$$
\begin{aligned}
& \frac{\partial \mathrm{Q}(\underset{\sim}{\mathrm{c}, \mathrm{d})}}{\partial \mathrm{c}}=\underset{\sim}{0} \\
& \Leftrightarrow-2 \mathrm{~V}^{\mathrm{T}} \mathrm{W}_{\text {het }}\left(\sigma^{2}\right) \mathrm{y}+2 \mathrm{~V}^{\mathrm{T}} \mathrm{W}_{\text {het }}\left(\sigma^{2}\right) \mathrm{T} \mathrm{d}+ \\
& 2\left(\mathrm{~V}^{\mathrm{T}} \mathrm{W}_{\text {het }}\left(\sigma_{\sim}^{2}\right) \mathrm{V}+\mathrm{N} \lambda \mathrm{V}\right) \mathrm{c}=0 \\
& \Leftrightarrow-\mathrm{W}_{\text {het }}\left(\sigma^{2}\right) \mathrm{y}+\mathrm{W}_{\text {het }}\left(\sigma^{2}\right) \mathrm{Td}+ \\
& {\left[\mathrm{W}_{\text {het }}\left(\sigma^{2}\right) \mathrm{V}+\mathrm{N} \lambda \mathrm{I}\right] \mathrm{c}=0} \\
& \text { If matrix } \mathrm{M}=\mathrm{W}_{\text {het }}\left(\sigma^{2}\right) \mathrm{V}+\mathrm{N} \lambda \mathrm{I} \text { then (25) gives }
\end{aligned}
$$

Eq. 26:

$\mathrm{Mc}=\mathrm{W}_{\text {het }}\left(\sigma_{\sim}^{2}\right)(\mathrm{y}-\mathrm{Td})$

If (26) is multiplied from the left hand side by $\mathrm{M}^{-1}$, it will give Eq. 27:

$$
\hat{\sim} \hat{\mathrm{c}}=\mathrm{M}^{-1} \mathrm{~W}_{\text {het }}\left(\sigma_{\sim}^{2}\right)(\mathrm{y}-\underset{\sim}{\mathrm{Td}})
$$

Furthermore, we also have partially derivative:

$$
\begin{aligned}
& \frac{\partial \mathrm{Q}(\underset{\sim}{\mathrm{c}, \mathrm{d})}}{\partial \mathrm{d}}=\underset{\sim}{0} \\
& \Leftrightarrow \mathrm{T}^{\mathrm{T}} \mathrm{W}_{\text {het }}\left(\sigma_{\sim}^{2}\right) \mathrm{y}+\mathrm{T}^{\mathrm{T}} \mathrm{W}_{\text {het }}\left(\sigma_{\sim}^{2}\right) \mathrm{T} \underset{\sim}{\mathrm{d}}+ \\
& \mathrm{T}^{\mathrm{T}} \mathrm{W}_{\text {het }}\left(\sigma^{2}\right) \mathrm{Vc}=0
\end{aligned}
$$

Because of (27), we obtain Eq. 28:

$$
\begin{aligned}
& -\mathrm{T}^{\mathrm{T}} \mathrm{W}_{\text {het }}\left(\underset{\sim}{\sigma^{2}}\right) \underset{\sim}{\mathrm{y}}+\mathrm{T}^{\mathrm{T}} \mathrm{W}_{\text {het }}\left(\underset{\sim}{\sigma^{2}}\right) \mathrm{T} \underset{\sim}{\mathrm{d}}+ \\
& \mathrm{T}^{\mathrm{T}}\left[\mathrm{W}_{\text {het }}\left(\underset{\sim}{\sigma^{2}}\right) \mathrm{VM}^{-1}\right] \mathrm{W}_{\text {het }}\left(\underset{\sim}{\sigma^{2}}\right)(\mathrm{y}-\mathrm{T} \underset{\sim}{\mathrm{T}})=\underset{\sim}{0}
\end{aligned}
$$

Since $\quad M=W_{\text {het }}\left(\sigma^{2}\right) V+N \lambda I \quad$ then $\mathrm{V}=\left[\mathrm{W}_{\text {het }}\left(\sigma_{\sim}^{2}\right)\right]^{-1}+(\mathrm{M}-\mathrm{N} \lambda \mathrm{I})$. Consequently, we have Eq. 29:

$$
\begin{aligned}
& \mathrm{VM}^{-1}=\left[\mathrm{W}_{\text {het }}\left(\sigma_{\sim}^{2}\right)\right]^{-1}+(\mathrm{M}-\mathrm{N} \lambda \mathrm{I}) \mathrm{M}^{-1} \\
& =\left[\mathrm{W}_{\text {het }}\left(\underset{\sim}{\sigma^{2}}\right)\right]^{-1}+\left(\mathrm{I}-\mathrm{N} \lambda \mathrm{M}^{-1}\right)
\end{aligned}
$$

If (29) is multiplied from the left hand side by $\mathrm{W}_{\mathrm{NS}}^{\mathrm{L}}\left(\underline{\sigma}^{2}\right)$, it will give Eq. 30: 
$\mathrm{W}_{\text {het }}\left(\sigma_{\sim}^{2}\right) \mathrm{VM}^{-1}=\mathrm{I}-\mathrm{N} \lambda \mathrm{M}^{-1}$

If we substitute (30) into (28), we get:

$$
\begin{aligned}
& -\mathrm{T}^{\mathrm{T}} \mathrm{W}_{\text {het }}\left(\sigma_{\sim}^{2}\right) \mathrm{y}+\mathrm{T}^{\mathrm{T}} \mathrm{W}_{\text {het }}\left(\sigma_{\sim}^{2}\right) \mathrm{Td}+ \\
& \left.\mathrm{T}^{\mathrm{T}}\left[\mathrm{I}-\mathrm{N} \lambda \mathrm{M}^{-1}\right] \mathrm{W}_{\text {het }}\left(\sigma_{\sim}^{2}\right)(\mathrm{y}-\mathrm{Td})=\underset{\sim}{\mathrm{d}}\right)
\end{aligned}
$$

Therefore, we obtain:

$$
\mathrm{N} \lambda \mathrm{T}^{\mathrm{T}} \mathrm{M}^{-1} \mathrm{~W}_{\text {het }}\left(\sigma_{\sim}^{2}\right) \mathrm{T} \underset{\sim}{\mathrm{d}}=\mathrm{N} \alpha \mathrm{T}^{\mathrm{T}} \mathrm{M}^{-1} \mathrm{~W}_{\text {het }}\left(\sigma_{\sim}^{2}\right) \mathrm{y}
$$

If two sides of the equation above is multiplied from the left hand side by $(\mathrm{N} \lambda)^{-1}$ and then by simplifying it, we will have Eq. 31:

$\hat{\mathrm{d}}=\left(\mathrm{T}^{\mathrm{T}} \mathrm{M}^{-1} \mathrm{~W}_{\text {het }}\left(\underset{\sim}{\sigma^{2}}\right) \mathrm{T}\right)^{-1} \mathrm{~T}^{\mathrm{T}} \mathrm{M}^{-1} \mathrm{~W}_{\text {het }}\left(\underset{\sim}{\sigma^{2}}\right) \mathrm{y}$

By substituting (31) into (27), it gives:

$\underline{\hat{\mathrm{c}}}=\mathrm{M}^{-1} \mathrm{~W}_{\text {het }}\left(\sigma_{\sim}^{2}\right)\left[\mathrm{I}-\mathrm{T}\left(\mathrm{T}^{\mathrm{T}} \mathrm{M}^{-1} \mathrm{~W}_{\text {het }}\left(\sigma_{\sim}^{2}\right) \mathrm{T}\right)^{-1} \times\right.$

$\left.\mathrm{T}^{\mathrm{T}} \mathrm{M}^{-1} \mathrm{~W}_{\text {het }}\left(\sigma^{2}\right)\right] \mathrm{y}$

Based on (31) and (32), we obtain weighted spline estimator for estimating heteroscedastic multi-response nonparametric regression curve that can be expressed as follows Eq. 33 and 34:

$\hat{\mathrm{f}}_{\lambda}=\left(\begin{array}{c}\hat{\mathrm{f}}_{1, \lambda_{1}} \\ \sim \sim \\ \hat{\mathrm{f}}_{2, \lambda_{2}} \\ \sim \\ \vdots \\ \underset{\sim}{\hat{\mathrm{f}}_{\mathrm{p}, \lambda_{\mathrm{p}}}}\end{array}\right)=\underset{\sim}{\sim} \underset{\sim}{\hat{\mathrm{d}}}+\underset{\sim}{\mathrm{c}}$

$=\left\{\mathrm{T}\left(\mathrm{T}^{\mathrm{T}} \mathrm{M}^{-1} \mathrm{~W}_{\text {het }}\left(\sigma_{\sim}^{2}\right) \mathrm{T}\right)^{-1} \mathrm{~T}^{\mathrm{T}} \mathrm{M}^{-1} \mathrm{~W}_{\text {het }}\left(\sigma_{\sim}^{2}\right)+\right.$

$\mathrm{VM}^{-1} \mathrm{~W}_{\text {het }}\left(\sigma_{\sim}^{2}\right)\left[\mathrm{I}-\mathrm{T}\left(\mathrm{T}^{\mathrm{T}} \mathrm{M}^{-1} \mathrm{~W}_{\text {het }}\left(\sigma_{\sim}^{2}\right) \mathrm{T}\right)^{-1} \times\right.$

$\left.\left.\mathrm{T}^{\mathrm{T}} \mathrm{M}^{-1} \mathrm{~W}_{\text {het }}\left(\sigma_{\sim}^{2}\right)\right]\right\} \mathrm{y}$

$=\mathrm{A}(\underset{\sim}{\lambda}) \mathrm{y}$

Where:

$$
\begin{aligned}
& \mathrm{A}(\underset{\sim}{\lambda})=\mathrm{T}\left(\mathrm{T}^{\mathrm{T}} \mathrm{M}^{-1} \mathrm{~W}_{\text {het }}\left(\underset{\sim}{\sigma^{2}}\right) \mathrm{T}\right)^{-1} \mathrm{~T}^{\mathrm{T}} \mathrm{M}^{-1} \times \\
& \mathrm{W}_{\text {het }}\left(\underset{\sim}{\sigma^{2}}\right)+\mathrm{VM}^{-1} \mathrm{~W}_{\text {het }}\left(\underset{\sim}{\sigma^{2}}\right) \times \\
& {\left[\mathrm{I}-\mathrm{T}\left(\mathrm { T } ^ { \mathrm { T } } \mathrm { M } ^ { - 1 } \mathrm { W } _ { \text { het } } \left({\underset{\sim}{\sigma}) \mathrm{T}}^{-1} \times\right.\right.\right.} \\
& \left.\mathrm{T}^{\mathrm{T}} \mathrm{M}^{-1} \mathrm{~W}_{\text {het }}\left(\sigma_{\sim}^{2}\right)\right]
\end{aligned}
$$

\section{DISCUSSION}

Estimating of nonparametric regression curve is the main problem in heteroscedastic multi-response nonparametric regression. For this objective, we determine weighted spline estimator by using Reproducing Kernel Hilbert Space (RKHS) approach to solve Penalized Weighted Least-Squares (PWLS) optimation (2). So that, we have weighted spline estimator $\hat{f}_{\lambda}$ as given in (33). Furthermore, the weighted spline estimator $\hat{\mathrm{f}}_{\lambda}$ has properties as follows:

- The weighted spline estimator $\hat{\mathrm{f}}_{\lambda}$ is linear in observation. This estimator is very useful to derive statistical inference for regression curve $\mathrm{f}$.

- The weighted spline estimator $\underset{\sim}{\hat{f}_{\lambda}}$ is a biased estimator for nonparametric regression curve $\mathrm{f}_{\sim}$. In other word, if we take expected value of (33), we will have:

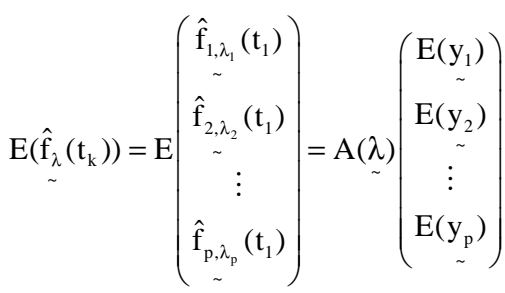

$$
\begin{aligned}
& =A(\lambda)\left(\begin{array}{c}
\mathrm{f}_{1}\left(\mathrm{t}_{1}\right) \\
\sim \\
\mathrm{f}_{2}\left(\mathrm{t}_{2}\right) \\
\sim \\
\vdots \\
\mathrm{f}_{\mathrm{p}}\left(\mathrm{t}_{\mathrm{p}}\right)
\end{array}\right) \neq\left(\begin{array}{c}
\mathrm{f}_{1}\left(\mathrm{t}_{1}\right) \\
\sim \\
\mathrm{f}_{2}\left(\mathrm{t}_{2}\right) \\
\sim \\
\vdots \\
\mathrm{f}_{\mathrm{p}}\left(\mathrm{t}_{\mathrm{p}}\right)
\end{array}\right)=\underset{\sim}{\sim}
\end{aligned}
$$

\section{CONCLUSION}

The reproducing kernel Hilbert space approach gives solution of penalized weighted least-squares optimation for estimating heteroscedastic multiresponse nonparametric regression curve which gives the weighted spline estimator. The estimator is a biased estimator for nonparametric regression curve. However, the estimator is linear in observation.

\section{ACKNOWLEDGEMENT}

Many thanks to the Directorate General of Higher Education, the Ministry of Education and Culture of the 
Republic of Indonesia passed through DP2M for the financial support of Doctoral Research Grant with contract number: 502/SP2H/PP/DP2M/VI/2010.

\section{REFERENCES}

Aydin, A., 2007. A comparison of the nonparametric regression models using smoothing spline and kernel regression. World Acad. Sci. Eng. Technol., 26: 730-734.

Cardot, H., C. Crambes, A. Kneip and P. Sarda, 2007. Smoothing splines estimators in functional linear regression with errors-in-variables. Computational Stat. Data Anal., 51: 4832-4848. DOI: 10.1016/j.csda.2006.07.029

Cox, D.D. and F. O'Sullivan, 1996. Penalized likelihood type estimators for generalized nonparametric regression. J. Mult. Anal., 56: 185206. DOI: $10.1006 /$ jmva. 1996.0010

Cox, D.D., 1983. Asymptotic for m-type smoothing spline. Annals Stat., 11: 530-551. DOI: 10.1214/aos/1176346159

Craven, P. and G. Wahba, 1979. Smoothing noisy data with spline function: Estimating the correct degree of smoothing by the method of generalized cross validation. Numer. Math., 31: 377-403.

Fernandez, F.M. and J.D. Opsomer, 2005. Smoothing parameter selection methods for nonparametric regression with spatially correlated errors. Canadian J. Stat., 33: 279-295. DOI: 10.1002/cjs.5550330208

Flessler, J.A., 1991. Nonparametric fixed-interval smoothing with vector splines. IEEE Trans. Signal Proc., 39: 852-859. DOI: 10.1109/78.80907

Gooijer, J.G.D., A. Gannoun and I. Larramendy, 1999. Nonparametric Regression with Serially Correlated Errors. 1st Edn., Tinbergen Institute, Rotterdam, pp: 19.

Kimeldorf, G. and G. Wahba, 1971. Some result on tchebycheffian spline functions. J. Math. Anal. Appli., 33: 82-95.

Koenker, R., N.G. Pin and S. Portnoy, 1994. Quantile smoothing splines. Biometrics, 81: 673-680. DOI: 10.1093/biomet/81.4.673

Lestari, B., I.N. Budiantara, S. Sunaryo and M. Mashuri, 2009. Spline estimator in homoscedastic multi-response nonparametric regression model. Proceedings of the Indonesian Mathmatics Sociaty International Conference on Mathematics and Its Application, Oct. 12-13, Yogyakarta-Indonesia.
Lestari, B., I.N. Budiantara, S. Sunaryo and M. Mashuri, 2010. Spline estimator in multi-response nonparametric regression model with unequal correlation of errors. J. Math. Stat., 6: 327-332. DOI: $10.3844 /$ jmssp. 2010.327 .332

Liu, A., T. Tong and Y. Wang, 2007. Smoothing spline estimation of variance functions. J. Comput. Graphical Stat., 16: 312-329. DOI: 10.1198/106186007X204528

Miller, J.J. and E.J. Wegman, 1987. Vector function estimation using splines. J. Royal Stat. Soc. Series B., 17: 173-180. DOI: 10.1016/03783758(87)90110-8

Oehlert, G.W., 1992. Relaxed boundary smoothing spline. Ann. Stat., 20: 146-160. DOI: 10.1214/aos/1176348516

Wahba, G., 1983. Bayesian confidence intervals for the cross-validated smoothing spline. J. Royal Stat. Soc. Series B., 45: 133-150.

Wahba, G., 1985. A comparison of GCV and GML for choosing the smoothing parameter in the generalized spline smoothing problem. Annals Stat., 13: 1378-1402. DOI: 10.1214/aos/1176349743

Wahba, G., 1990. Spline Models for Observational Data. 1st Edn., SIAM, Philadelphia, ISBN-10: 0898712440, pp: 180.

Wahba, G., 1992. Multivariate functional and operator estimation, based on smoothing spline and reproducing kernels. Department of Statistics.

Wahba, G., 2000. An introduction to model building with reproducing kernel hilbert spaces. Department of Statistics, University of Wisconsin, Madison.

Wang, Y., 1998. Smoothing spline models with correlated random errors. J. Am. Stat. Assoc., 93: 341-348.

Wang, Y., W. Guo and W.B. Brown, 2000. Spline smoothing for bivariate data with applications to association between hormones. Stat. Sinica, 10: 377-397.

Wegman, E.J., 1981. Vector spline and estimation of filter function. Technometrics, 23: 83-89. 